\title{
Synthesis and Characteristic of Novel Soluble Triazoleimide Oligomers with Terminated Arylacetylene
}

\author{
Xiao'an Zhou, Lei Du, Liqiang Wan, Xiaofei Wang, Yanpeng E, and Farong Huang* \\ Key Laboratory for Specially Functional Polymeric Materials and Related Technology of the Ministry of Education, \\ School of Materials Science and Engineering, East China University of Science and Technology, Shanghai 200237, \\ P. R. China. *E-mail: fhuanglab@ecust.edu.cn \\ Received May 24, 2010, Accepted July 26, 2010
}

\begin{abstract}
Novel soluble triazoleimide oligomers terminated with arylacetylene terminated were synthesized by the $\mathrm{Cu}(\mathrm{I})$-catalysed 1,3-dipolar cycloaddition polymerization of diazides and imide-containing dialkyne. Several molecular weight triazoleimide oligomers were prepared from diazide and dialkyne monomers with different stoichiometric combinations. The curing behaviors of the oligomers were tested by differential scanning calorimetry (DSC). The thermal properties of the cured products were evaluated by DSC and thermogravimetric analysis (TGA). These cured oligomers showed the glass transition temperature of about $225-235^{\circ} \mathrm{C}$ and the decomposition temperature (at $5 \%$ weight loss) of about $385-393{ }^{\circ} \mathrm{C}$ in nitrogen.
\end{abstract}

Key Words: Triazoleimide, Oligomers, Arylacetylene

\section{Introduction}

Polyimides have been extensively utilized owing to their excellent thermal stability, mechanical properties, good chemical resistance, high dimensional stability and good electric properties. ${ }^{1}$ However, the poor processability limited their widespread application. To improve their processability (melt processing properties or solubility in organic solvents), more and more attention has been focused on the study of various kinds of PETIs ${ }^{2-5}$ (phenylethynyl-terminated polyimide oligomers). These resins are general prepared by using end-capping reagent.

$\mathrm{The} \mathrm{Cu}(\mathrm{I})$-catalyzed cycloaddition of azides and alkynes is known as the "cream of the crop" of the click chemistry. ${ }^{6,7}$ It has been utilized in material synthesis and a wide range of polymer architectures have been designed and made by the CuAAC. ${ }^{8-14}$ Material scientists not only use it as a synthetic tool but also discover applications for the triazole moiety. Sharpless, Fokin, and Finn combined di-, tri-, and tetravalent azides and alkynes to produce crosslinked polymers as adhesives, which could bind metal surfaces together. ${ }^{15}$ The triazole's electronic properties have made it popular among fluorgenic probes. Fahrni and Wang both demonstrated a dye that exhibited no fluorescene until click chemistry was performed to yield a triazole moiety. ${ }^{16}$ The step-growth polymerization of diazide and dialkyne has been used to prepare a series of functionalized polyesters. ${ }^{17,18}$

1,2,3-Triazoles are noteworthy for their pseudoaromatic nature, large dipole moment, and excellent hydrogen-bonding ability. ${ }^{31}$ It has good affinity for metallic surfaces. We report the synthesis and characterization of a kind of novel soluble arylacetylene-terminated triazoleimide oligomers by the CuAAC step-growth polymerization of diazides and dialkyne in solution in this paper. A series of $t$ triazoleimide oligomers with various molecular weigh were prepared from dialkyne and diazide monomer with different stoichiometries. The curing behave of the oligomers were tested by differential scanning calorimetry (DSC). The properties of the cured products of the oligomers were also investigated.

\section{Experimental Part}

Materials. 2,2-Bis [4-(3,4-dicarboxyphenoxy) phenyl] propane dianhydride (BPADA) was used as purchased from Shanghai Synthesis Resin Institute. 3-Amine-phenylethyne (APE), tetrahydrofuran (THF), acetone, dimethylacte (DMAc), dimethylformide (DMF), toluene, $N$-methyl-2-pyrrolidinone (NMP) were purchased and used as received (from Shanghai No.1 Reagent Company). $p$-Xylylene diazide (XDA) was synthesized in our laboratory according to the method described in the literature. ${ }^{19-22} \mathrm{XDA}: \mathrm{mp} 27-29^{\circ} \mathrm{C}$ (Reported value: 27.5 $\left.29^{\circ} \mathrm{C}\right),{ }^{32}$ FT-IR: $2098 \mathrm{~cm}^{-1}$ (azide), ${ }^{1} \mathrm{H}-\mathrm{NMR}:\left(\mathrm{CDCl}_{3}, \mathrm{TMS}\right)$ : 7.33 (s, 4H, Ar-H), 4.35 (s, $4 \mathrm{H}, \mathrm{Ar}-\mathrm{CH}_{2} \mathrm{~N}_{3}$ ). The spectroscopic data was consistent with reported value.

Measurements. ${ }^{13} \mathrm{C}-\mathrm{NMR}$ spectra $(125 \mathrm{MHz})$ and ${ }^{1} \mathrm{H}-\mathrm{NMR}$ $(500 \mathrm{MHz})$ were recorded on a Bruker AVANCE 500 Spectrometer in DMSO. FT-IR spectra were determined by a Bio-Rad Digilab Division FTS-165 Spectrometer. Differential scanning calorimeter (DSC) analyses were performed with UniversalV2.3 TA Instruments with 2910 modulated system. The heating rate was $10^{\circ} \mathrm{C} / \mathrm{min}$ at a flow rate of nitrogen $15 \mathrm{~cm}^{3} / \mathrm{min}$. Thermogravimetric analysis (TGA) was performed on a METTLER TGA/SDTA 851 analyzer with a heating rate of $10^{\circ} \mathrm{C} / \mathrm{min}$. Elemental analyses were determined with a Elementar Analysensysteme $\mathrm{GmbH}$ vario EL III analyzer.

Synthesis of 3,3-bis ( $N$-acetylenephenylphthalimide) ether of bisphenol A(BAPIPA). The synthesis of 3,3-bis ( $N$-acetylenephenylphthalimide) ether of bisphenol A (BAPIPA) is shown in Scheme 1. In a $250 \mathrm{~mL}$ three-necked flask equipped with a refluxing condenser and a stirrer, $4 \mathrm{mmol}$ of BPADA, $8 \mathrm{mmol}$ 3-amine-phenylacetylene and $50 \mathrm{~mL}$ acetone were charged. The solution was formed after stirring for a few minutes. The solution was heated to refluxing. The reaction continued for $6 \mathrm{~h}$. Then $50 \mathrm{~mL}$ acetic anhydride and $70 \mathrm{~mL}$ triethylamine as catalyst were added. The temperature of the reaction was increased to $80^{\circ} \mathrm{C}$ and maintained for $4 \mathrm{~h}$. The product was precipitated in solvent. The crude product was collected by filtration, washed 
<smiles>CC#Cc1cccc(NC[NH+]2OC(=O)c3ccc(Oc4ccc(C(C)(C)c5ccc(Oc6ccc7oc(=O)oc(=O)c7c6)cc5)cc4)cc3C2=O)c1</smiles>

Scheme 1. Synthesis of the phthalimide dialkyne monomer BAPIPA

several times by acetone and dried at $80{ }^{\circ} \mathrm{C}$ for $24 \mathrm{~h}$ in a vacuum oven.

Yield: 90\%. FT-IR $\left(\mathrm{cm}^{-1}\right)$ : 3276 ( $\equiv \mathrm{C}-\mathrm{H}$ stretch), $2103(\mathrm{C} \equiv \mathrm{C})$, $1774(\mathrm{C}=\mathrm{O}$ antisym stretch $), 1719(\mathrm{C}=\mathrm{O}$ sym stretch, imide $\mathrm{I})$, 1376 (C-N-C, imide II).

${ }^{1} \mathrm{H}-\mathrm{NMR}\left(500 \mathrm{MHz}, \mathrm{DMSO}-d_{6}, \mathrm{ppm}\right): \delta 1.72\left(\mathrm{~s}, 6 \mathrm{H},-\mathrm{CH}_{3}\right)$, $4.31(\mathrm{~s}, 2 \mathrm{H},-\mathrm{C} \equiv \mathrm{C}-\mathrm{H}), 7.12-7.98\left(\mathrm{~m}, 22 \mathrm{H},-\mathrm{C}_{6} \mathrm{H}_{4}-,-\mathrm{C}_{6} \mathrm{H}_{3}\right.$ and $\left.-\mathrm{C}_{6} \mathrm{H}_{4}\right) \mathrm{ppm} .{ }^{13} \mathrm{C}$ NMR (125 MHz, DMSO- $\left.d_{6}, \mathrm{ppm}\right): \delta 166.0$ $(\mathrm{N}-\mathrm{C}=\mathrm{O}), 162.8,132.1,129.3,128.6,122.8,111.5\left(-\mathrm{C}_{6} \mathrm{H}_{3}-\right)$; 152.4, 147.1, 130.1, 119.7 (O- $\left.\mathrm{C}_{6} \mathrm{H}_{4}-\right)$; 134.1, 131.1, 130.1, 127.8, $125.9,125.1\left(-\mathrm{C}_{6} \mathrm{H}_{4}-\mathrm{C} \equiv \mathrm{C}-\mathrm{H}\right), 82.5(-\mathrm{C} \equiv \mathrm{CH}), 81.7(-\mathrm{C} \equiv \mathrm{CH})$, $42.0\left(-\mathrm{C}\left(\mathrm{CH}_{3}\right)_{2}-\right), 30.5\left(-\mathrm{CH}_{3}\right)$.

Element analysis. Calcd. for $\mathrm{C}_{47} \mathrm{H}_{30} \mathrm{~N}_{2} \mathrm{O}_{6}: \mathrm{C}, 78.55 \%$; $\mathrm{N}$, $3.90 \%$; H, 4.18\%. Found: C, 78.10\%; N, 3.82\%; H, 4.16\%.

Synthesis of arylacetylene-terminated imide oligomers. The oligomers were prepared by the $\mathrm{Cu}(\mathrm{I})$-catalyzed 1,3-dipolar cycloaddition step-growth polymerization. A typical procedure for the preparation was as follows: To a $100 \mathrm{~mL}$ three-neck round-bottom flask equipped with a magnetic stirrer, a thermometer and a condenser were charged $10 \mathrm{mmol}$ BAPIPA, $6 \mathrm{mmol}$ $\mathrm{XDA}$ and $50 \mathrm{~mL}$ NMP. The temperature of the mixture was increased to $60{ }^{\circ} \mathrm{C}$ under stirring. Then sodium ascorbate $(0.198 \mathrm{~g}, 1 \mathrm{mmol})$ and $\mathrm{CuSO}_{4} 5 \mathrm{H}_{2} \mathrm{O}(0.125 \mathrm{~g}, 0.5 \mathrm{mmol})$ were added into the flask. After the mixture stirred for 5 minutes, $10 \mathrm{mmol}$ triethylamine was added. The solution stirred at $60{ }^{\circ} \mathrm{C}$ for $4 \mathrm{~h}$ to preduce oligomers solution. Then the solution was poured into $1000 \mathrm{~mL}$ of water. The precipitation was collected and washed by water. Then the dried precipitation was dissolved in NMP and precipitated in water again. The precipitation product was dried at $100{ }^{\circ} \mathrm{C}$ under vacuum for $24 \mathrm{~h}$. As a result, the corresponding oligomer was obtained. Similarly, with changing the molar ratios of BAPIPA to XDA with 5:4, 8:7 and 1:1, a series of arylacetylene-terminated oligomers with different molecular weight were prepared. The oligomers were obtained as light white solids.

\section{Results and Discussion}

Synthesis and characterization of Arylacetylene-terminated imide oligomers. The arylacetylene-terminated tiazoleimide<smiles>C#Cc1cccc(N2C(=O)c3ccc(Oc4ccc(C(C)(C)c5ccc(Oc6ccc7c(c6)C(=O)N(c6cccc(C#C)c6)C7=O)cc5)cc4)cc3C2=O)c1</smiles>

oligomers were prepared from 3,3-bis ( $N$-acetylenephenylphthalimide) ether of bisphenol A and the diazides (BAPIPA and XDA) by 1,3-dipolor cycloaddition polymerizations in NMP as shown in Scheme 2. Click polymerization of dialkyne with diazides were carried out under mild conditions. The polymerisation reactions were performed by mixing different molar ratios of dialkyne with diazides in NMP at $60^{\circ} \mathrm{C}$ under an open atmosphere.

The chemical structures of the obtained arylacetylene-terminated triazoleimide oligomers were characterized by FT-IR spectroscopy and ${ }^{1} \mathrm{H}-\mathrm{NMR}$. The IR spectra of oligomers derived from BAPIPA and XDA were given in Figure 1. The imide characteristic bands at $1780,1720 \mathrm{~cm}^{-1}$ and $1370 \mathrm{~cm}^{-1}$ had no change before and after the polymerization, which implied that polymerization did not affect the imide units. The absorption bands associated with the triazole appeared at $3120 \mathrm{~cm}^{-1}$. The absorption bands associated with the vibrational stretching of $\equiv \mathrm{C}-\mathrm{H}$ at $3247 \mathrm{~cm}^{-1}$ and $\mathrm{C} \equiv \mathrm{C}$ at $2009 \mathrm{~cm}^{-1}$ become weak with the molar ratios of BAPIPA to XDA increasing.

The ${ }^{1} \mathrm{H}-\mathrm{NMR}$ spectroscopy of oligomers showed the presence of the expected repeating unit. Since the ${ }^{1} \mathrm{H}-\mathrm{NMR}$ spectrum of oligomers were similar, thus only the spectroscopies of oligo-

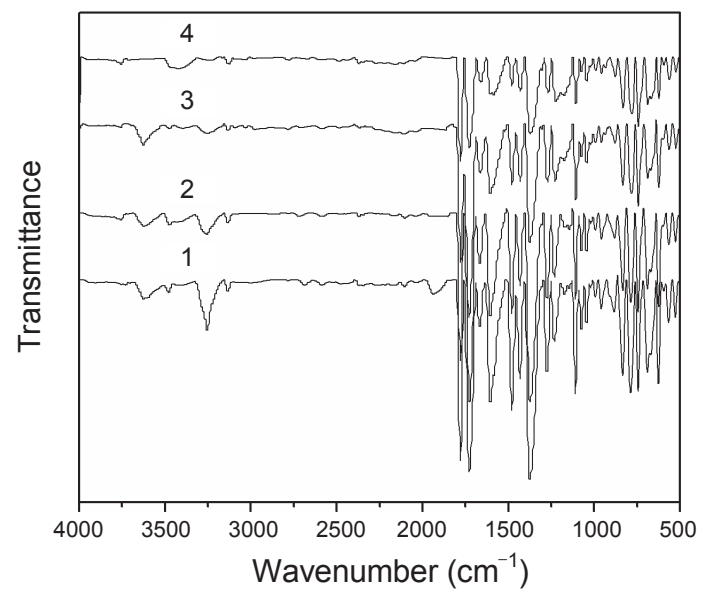

Figure 1. FT-IR spectra of the oligomers; Molar ratios of BAPIPA to XDA: 1-3:2, 2-5:4, 3-8:7, 4-1:1.<smiles></smiles>

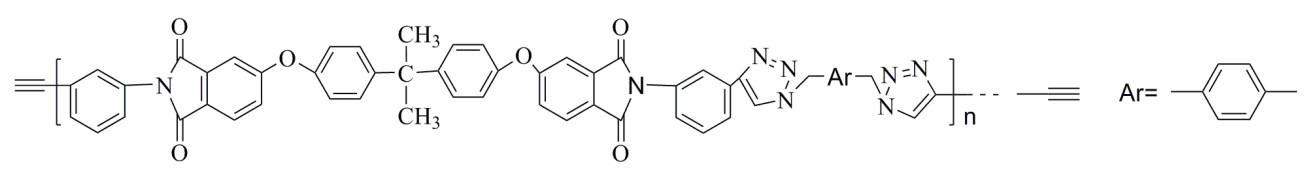

Scheme 2. Synthesis of arylacetylene-terminated imide oligomers by CuAAC step-growth polymerization 

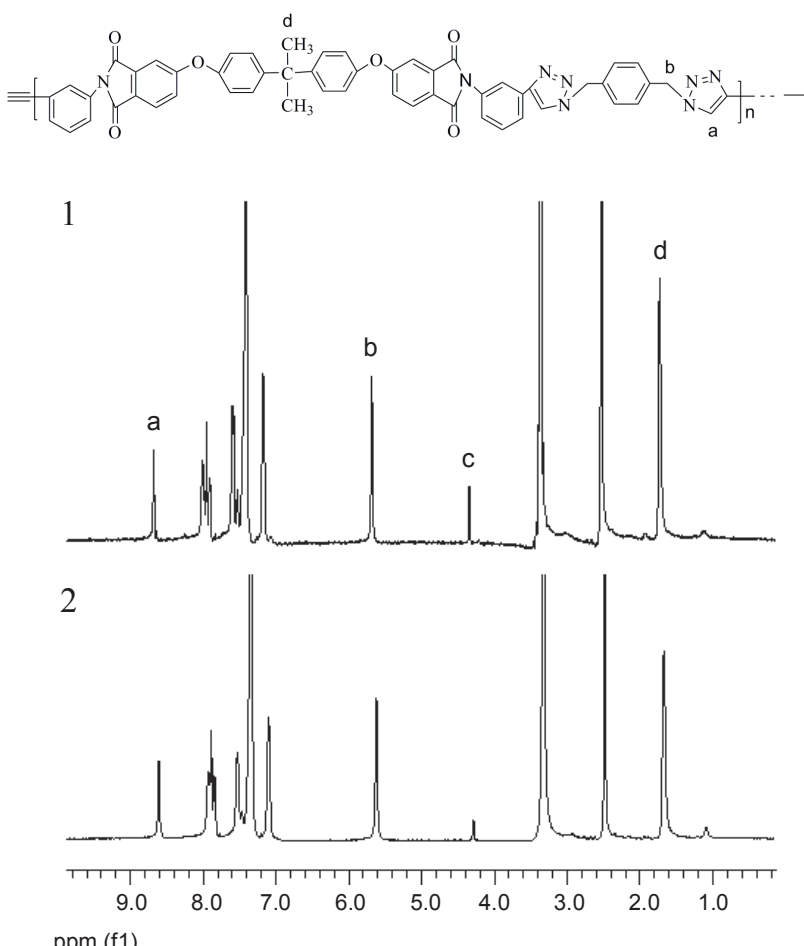

Figure 2. ${ }^{1} \mathrm{H}-\mathrm{NMR}$ spectroscopy of oligomers derived from BAPIPA and XDA; Molar ratios of BAPIPA to XDA: 1 - 3:2, 2 - 8:7.

mers derived from BAPIPA and XDA with molar ratios of 3:2 and 8:7 were showed in Figure 2. The methylene protons adjacent to the azide group of XDA resonated at $\delta 4.71$; this peak completely disappeared after polymerization. The acetylene protons of BAPIPA resonated at $\delta 4.31$ decreased apparently. The new resonance peaks were observed at $\delta 8.65,5.63$. The peak at 8.65 was associated with the resonances of 1,2,3-triazole rings (a). These indicated that the azide groups of the monomers had undergone the polycycloaddition reactions to form the triazole rings in the oligomers The peaks at 5.63(c) are due to the downfield shift of the benzyl proton resonances of XDA after the polymerization reaction. The absorption peaks at 7.2 $8.0 \mathrm{ppm}$ were assigned to the aromatic protons in the polytriazoleimides backbone. The integrals of resonance peaks a and $\mathrm{c}$ in Figure 2 were consistent with the structures of the oligomers (the same as molar ratios of BAPIPA to XDA).

The novel triazoleimide oligomers could dissolve in most

Table 1. Solubility and inherent viscosities $\left(\eta_{\text {inh }}\right)$ data of polytriazoleimides

\begin{tabular}{ccccccc}
\hline \multirow{2}{*}{ Oligomers } & \multicolumn{7}{c}{ Solvent } \\
\cline { 2 - 7 } & DMF & NMP & DMSO & $\mathrm{CHCl}_{3}$ & THF & Acetone \\
\hline 1 & ++ & ++ & ++ & + & + & - \\
2 & ++ & ++ & ++ & + & + & - \\
3 & ++ & ++ & ++ & + & + & - \\
4 & ++ & ++ & ++ & - & - & - \\
\hline
\end{tabular}

Oligomers derived from BAPIPA and XDA; Molar ratios of BAPIPA to XDA: 1 - 3:2, 2 - 5:4, 3 - 8:7, 4 - 1:1. + + soluble at room temperature; + soluble on heating; - insoluble even on heating.

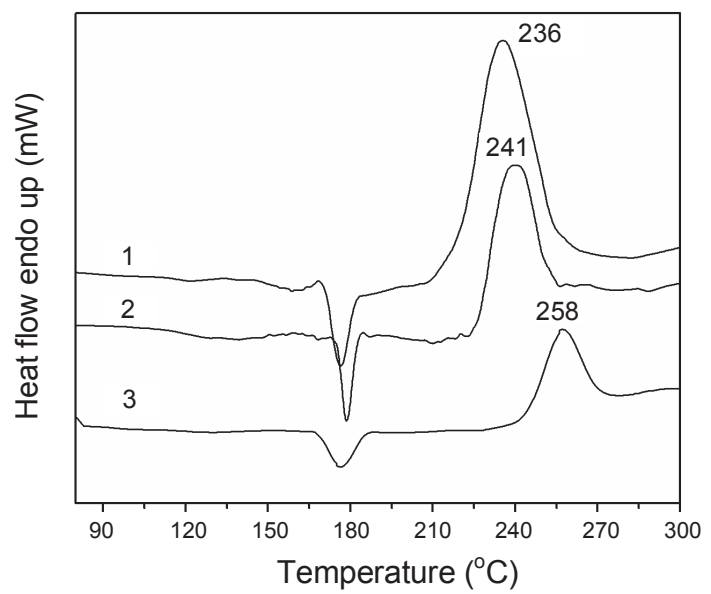

Figure 3. DSC curve of the oligomers derived from BAPIPA and XDA; Molar ratios of BAPIPA to XDA: 1 - 3:2, 2 - 5:4, 3 - 8:7.

of organic polar solvents. The solubility of the oligomers was determined by dissolving $0.3 \mathrm{~g}$ of oligomers in $10 \mathrm{~mL}$ of solvent, as shown in Table 1. It could be seen that oligomers with different molecular weight could be dissolved in most of organic polar solvents, such as DMSO, NMP and DMAc, even at room temperature in most cases. The result indicated that the oligomers containing 1,2,3-triazole rings and imide units were dissolved readily in most of organic polar solvents at room temperature.

Thermal behaviors of arylacetylene-terminated triazoleimide oligomers and their thermal properties. The curing behaves of the arylacetylene-terminated triazoleimide oligomers were measured by DSC under nitrogen. The DSC curves of oligomers derived from BAPIPA and XDA were shown in Figure 3. These results are also summarized in Table 2 . There were an endothermic peak and an exothermic one in the curves. The exothermic peaks were attributed to a cross-linking reaction of the arylacetylene groups, which showed the enthalpy of $66.8-97.4$ $\mathrm{J} / \mathrm{g}$ and a onset temperature of $215-240{ }^{\circ} \mathrm{C}$. With the increasing of molar ratios of BAPIPA to XDA, the onset and peak temperature increased.

The cure process of the arylacetylene group has been widely investigated. ${ }^{23-28}$ Solid-state ${ }^{13}$ C-NMR, FT-IR and ESR analyses have brought some insight into the cure mechanism of arylacetylene group. The results demonstrated that the arylacetylene group underwent cyclotrimerization, hydrogen addition, DielsAlder cycloaddition, etc., and formed a complex crosslinked structure like condensed polycyclic aromatic structure during the cross-linking process.

The oligomers were weighted and kept in an oven at $200{ }^{\circ} \mathrm{C} /$

Table 2. Thermal and tensile properties of the polytriazoleimides

\begin{tabular}{ccc}
\hline Oligomers & $T_{\text {exo }}\left({ }^{\circ} \mathrm{C}\right)$ & $\Delta \mathrm{H}\left(\mathrm{J} \mathrm{g}^{-1}\right)$ \\
\hline 1 & 236 & 97.4 \\
2 & 241 & 85.4 \\
3 & 258 & 66.8 \\
\hline
\end{tabular}

Oligomers derived from BAPIPA and XDA; Molar ratios of BAPIPA to XDA: 1 - 3:2, 2 - 5:4, 3 - 8:7. 
Table 3. Thermal properties of the cured oligomers

\begin{tabular}{cccc}
\hline Cured oligomers & $T_{g}$ & $T_{d 5}$ & Char Yield $^{a}(\%)$ \\
\hline 1 & 225 & 393 & 60 \\
2 & 229 & 385 & 51 \\
3 & 235 & 390 & 48 \\
\hline
\end{tabular}

Oligomers derived from BAPIPA and XDA; Molar ratios of BAPIPA to XDA: 1 - 3:2, 2 - 5:4, 3 - 8:7. ${ }^{a}$ Residual weight (\%) when the polymers were heated to $800{ }^{\circ} \mathrm{C}$ in nitrogen.

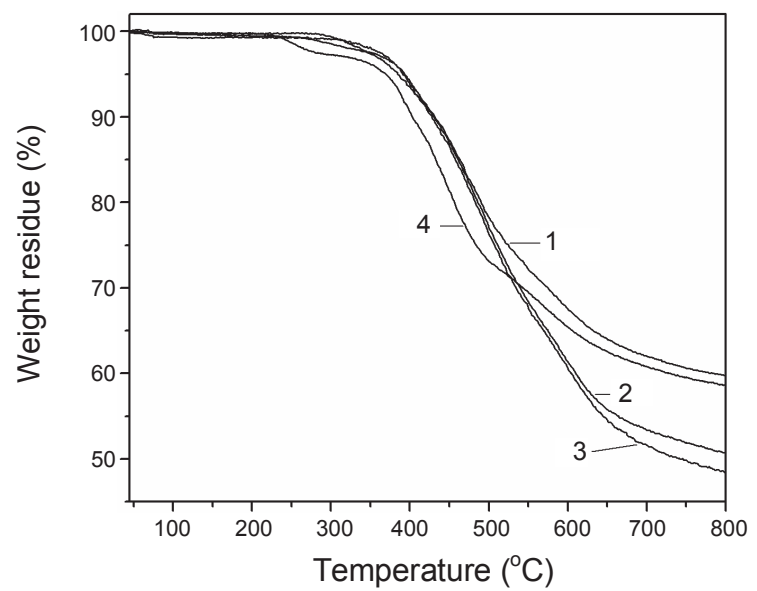

Figure 4. The TGA curves of oligomers derived from BAPIPA and XDA; Molar ratios of BAPIPA to XDA: 1 - 3:2, 2 - 5:4, 3 - 8:1, 4 - 1:1.

$2 \mathrm{~h}+260{ }^{\circ} \mathrm{C} / 4 \mathrm{~h}$ to get cured products under air. The thermal properties of the cured oligomers were determined by using DSC and TGA. The results were showed in Table 3 . The values of glass transition temperature $(\mathrm{Tg})$ of these cured oligomers were tested by DSC with heating rate of $20^{\circ} \mathrm{C} / \mathrm{min}$. These cured oligomers showed $\mathrm{Tg}$ 's of $225-235^{\circ} \mathrm{C}$.

The TGA curves of cured oligomers were shown in Figure 4. The decomposition temperatures of the cured oligomers at $5 \%$ weight loss were between 370 to $395^{\circ} \mathrm{C}$ in nitrogen. The decomposition temperatures of the cured oligomers derived from BAPIPA and XDA were also between 375 to $395^{\circ} \mathrm{C}$ in nitrogen. These illustrated the cured products were thermally stable. As compared with conventional polyimides, ${ }^{29,30}$ the cured oligomers showed lower decomposition temperature due to the weak bonding of the -C-N- adjacent to diazide, and the easy breakage during the heating process.

\section{Conclusion}

In this paper, we have prepared various linear arylacetyleneterminated triazoleimide oligomers by the $\mathrm{CuAAC}$ polymerization of a diazide and dialkyne in solution. These oligomers were characterized by FT-IR and ${ }^{1} \mathrm{H}$ NMR. The novel arylacetylene-terminated triazoleimide oligomers showed good solubility in organic polar solvents. The curing behavior of the oligomers were tested by DSC. The oligomers showed a low onset curing temperature of $215-240{ }^{\circ} \mathrm{C}$. The cured oligomers showed high thermal stability and high glass transition temperature. The good properties of triazoleimide oligomers could be utilized in high-performance metal coatings, matrix and adhesives. The adhesion and anti-corrosin functionalities and applications of the materials are currently under investigations.

Acknowledgments. The authors gratefully acknowledge the financial support of the National High Technology Research and Development Program of China (Grant No. 2002A305103) and the National Basic Research Program of China (Grant No. 5131101).

\section{References}

1. Wilson, D.; Stenzenberger, H. D.; Hergenrother, P. M. Polyimides; Blackie: New York, USA, 1990; pp 4-57.

2. Jayaraman, S.; Srinivasan, R.; McGrath, J. E. J. Polym. Sci. Part A: Polm. Chem. 1995, 33, 1551.

3. Bryant, R. G.; Jensen, B. J.; Hergenrother, P. M. J. Appl. Polym. Sci. 1996, 59. 1249.

4. Meyer, G. W.; Pak, S. J.; Lee, Y. J.; McGrath, J. E. Polymer 1995, 11, 2303.

5. P Hergenrother, M. J.; Connell, W.; Smith, J. G. Polymer 2000 , 41,5073

6. Kolb, H. C.; Finn, M. G.; Sharpless, K. B. Angew. Chem. Int. Ed. 2001, 40, 2004.

7. Rostovtsev, V. V.; Green, L. G.; Fokin, V. V.; Sharpless. K. B. Angew. Chem. Int. Ed. 2002, 41, 2596.

8. Helms, B.; Mynar, J. L.; Hawker, C. J.; Fréchet, J. M. J. Am. Chem. Soc. 2004, 126, 15020.

9. Hawker, C. J.; Wooley, K. L. Science 2005, 309, 1200.

10. Fournier, D.; Hoogenboom, R.; Schubert, U. S. Chem. Soc. Rev. 2007, 36, 1369.

11. Nandivada, H.; Lahann, J.; Jiang, X. Adv. Mater. 2007, 19, 2197.

12. Wolfgang, H. B.; Robert, S. Macromol. Rapid Commun. 2007, 28,15 .

13. Lundberg, P.; Hawker, C. J.; Hult, A.; Malkoch, M. Macromol. Rapid Commun. 2008, 29, 998.

14. Meldal, M. Macromol. Rapid Commun. 2008, 29, 1016.

15. Diaz, D. D.; Punna, S.; Holzer, P.; Mcpherson, A. K.; Sharpless, K. B.; Fokin,V. V.; FINN, M. G. J. Polym. Sci. Part A: Polym. Chem. 2004, 42, 4392.

16. Gallardo, H.; Ely, F.; Bortoluzzi, A.; Conte J. G. Liquid Crystals 2005, 32, 667.

17. Nagao, Y.; Takasu, A. Macromol. Rapid Communications 2009 , 30, 199.

18. Billiet, L.; Fournier, D.; Prez, F. D. J. Polym. Sci. Part A: Polym. Chem. 2008, 46, 6552.

19. Wang, X. F.; Zhao, Z. F.; Tian, J. J.; Huang, F. R.; Du, L. Polym. J. 2009, 41, 498 .

20. Wan, L. Q.; Luo,Y. H.; Xue, L.; Tian, J. J.; Hu, Y. H.; Qi, H. M.; Shen, X. N.; Huang, F. R.; Du, L.; Chen, X. B. J. Appl. Polym. Sci. 2007, 104, 1038.

21. Tian, J. J.; Wan, L. Q.; Huang, J. Z.; Hu, Y. H.; Huang, F. R.; Du, L. Polym. Adv. Technol. 2007, 18, 556.

22. Hu, Y. H.; Luo, Y. H.; Wan, L. Q.; Qi, H. M.; Huang, F. R.; Du, L. Acta Polymerica Sinica 2005, 4, 560.

23. Fang, X.; Rogers, D. F.; Scola, D. A.; Stevens, M. P. J. Polym. Sci. Polym. Chem. 1998, 36, 461.

24. Fang, X.; Simone, C. D.; Stevens, M. P. D.; Scola, A. Macromolecules 2000, 33, 1671.

25. Swanson, S. A.; Fleming, W. W.; Hofer, D. C. Macromolecules 1992, 25, 582 .

26. Nakamura, K.; Ando, S. Polymer 2001, 42, 4045.

27. Shigeki, K.; Okita, K. Macromolecules 1998, 31, 2804.

28. Kim, Y. H.; Kim, H. S.; Kwon, S. K. Macromolecules 2005, 38 , 7950.

29. Liaw, D. J.; Liaw, B. Y. Chem. Mater. 1998, 10, 734

30. Abboud, J. M.; Foces-Foces, C.; Notario, R.; Trifonov, R. E. Eur. J. Org. Chem. 2001, 16, 3013. 